\title{
"I'll bet on me! group psychotherapeutic treatment for gambling disorder"
}

\begin{abstract}
An alarming fact of the last years is the emergence of new pathologies of abuse behaviour. Among the main "new addictions", Gambling disorder has been included by the DSM-5 in the category of Dependences, more specifically in the sub-category of "Non-substance use disorders". When desire to gamble is so intense and uncontrollable that it converts into the impossibility to escape it, we can talk about "craving". Craving is the pathological version of desire: desire that no longer corresponds to liking and leads to dependence. In technical terms, we say that dependence occurs when "liking" no longer keeps pace with "wanting" because wanting goes on autonomously. On this point, the territorial Service for the Treatment of Drug Addiction of Ostia Lido, Rome, Local Health Authority (ASL) Roma D, has proposed a psychotherapeutic intervention based on group treatment with people suffering from gambling disorder, aimed at increasing the awareness of their psychic functioning, recognizing and managing desire and impulse to gamble, favouring change, recognizing risks and obstacles, developing personal defence strategies, increasing problem-solving capacities, monitoring and assessing progresses and changes.
\end{abstract}

Keywords: non-substance addictions, new addictions, gambling disorder, behavioural addictions, craving, group psychotherapeutic treatment
Volume 7 Issue 4 - 2019

\section{Francesca Romana Ficorilli \\ Psychologist/Psychotherapist, Clinical criminologist, Psycho- diagnostician, Legal and forensic psychologist, Italy}

Correspondence: Francesca Romana Ficorilli, Psychologist/ Psychotherapist, Clinical criminologist, Psycho-diagnostician, Legal and forensic psychologist, Service for the Treatment of Drug Addiction (Ser.T) Ostia Lido, Rome, Local Health Authority (ASL) Roma D, Italy,

Email francescaromana.ficorilli@gmail.com

Received: July II, 2019 | Published: July 19, 2019

\section{Introduction}

The phenomenon of gambling has reached alarming levels also in Italy. This is favoured by a two-sided State that, on the one hand, introduces new offers of public gambling every year and, on the other hand, funds campaigns aimed at de-addiction. The market of gambling knows no crisis, in spite of the "recession" period faced by Italy, so much that it is among the countries most affected by the issue. The Independent Authority for the Administration of State Monopolies (AAMS) says that gambling expenses increased from Euro 14.3 billion in 2000 to over Euro 101.8 billion in 2017.

\section{Material and method}

The group treatment for compulsive gambling in a group of participants composed of a minimum of 5 to a maximum of 10 people (average age 45years) consisted of a first cycle of 20 fortnightly sessions of 90min. each (from May 2010 to February 2011). On an experimental basis, this was followed by a second cycle of sessions (from March to July 2011) where, changing the group structure and making it more open, it was allowed to add new people. Finally, follow-up monthly meetings, over a period of 18 months, allowed monitoring the group therapy outcome, including, at the request of the patient, individual support and/or support to their family. The treatment was co-performed by two psychologists at the premises.

\section{Outcome}

At the end of the second cycle of meetings with the therapeutic group (March 2011), 5 patients out of a stabilized number of 8 reached abstinence from gambling. The treatment tried to increase the awareness of their psychic functioning in order to identity weaknesses and resources through different aspects:

i. Confrontation among participants and compliance with the group's rules ii. Telling of experiences and personal problems

iii. Self-examination of one's own thoughts, emotions and reactions in the different moments relating to gambling-related situations

iv. The range of alternatives to consider as a substitute for gambling

\section{Conclusions}

The experience of the group treatment, by creating a temporary group identification, allows individuals to get from the group an identity share that the gambling disorder itself contributes to spread. This way, the aware use of planned strategies and the sharing of common issues make it possible to use the group treatment for compulsive gambling. This kind of treatment appears to be one of the most effective resources the State may and shall invest in so that Ser. Ts become an increasingly visible point of reference for the treatment and cure of "new" "non-substance use" disorders.

\section{Text}

The rampant social phenomenon of pathological gambling in the last ten years, favoured by a growing market that offers several increasingly more legalized types of games, seems to lead to a new class of abuse behaviour disorders. Amongst them Gambling Disorder that the DSM-5 classifies into the category of Dependencies, more specifically in the sub-category of "Non-substance use disorders". The peculiar characteristic of this affection is a persistent, recurring and maladaptive gambling behaviour that affects personal, familiar and working activities, with a compulsion to spend increasing amounts of time and money in gambling activities, entrapping the gambler in a self-destructive dynamic. The passage from "desire" to gamble to "compulsion" to gamble occurs when desire no longer corresponds to liking; does not presuppose it, no longer evokes it, does not extinguish with its satisfaction and does not cease with the lack of liking. In technical terms, we say that dependence occurs 
when "liking" no longer keeps pace with "wanting" because wanting goes on autonomously. In this case, "wanting" becomes "craving" (pathological desire) and compulsion to gamble, which is lived in a pervasive way, becomes compulsive gambling. A desire, which is no longer controlled and regulated based on liking, causes a sense of void and sufferance in the gambler, as well as a series of problematic consequences in their life. This work aims at investigating how the group therapeutic treatment for gambling disorder acts on the cognitive restructuring of the gambler's dynamics and represents a first line treatment for these new behavioural dependences.

A key condition is the perceived support with the sharing of one's own experiences. The programme provides for the increasing of the awareness of one's own psychic behaviour by working-through cognitive distortions within the therapeutic group. This provides support for the development of more adaptive problem-solving strategies and abilities, but first of all it represents an effective containment of dysregulated emotions that is able to promote personal change. Gambling, with its seesaw "win-loss" trend, concretizes the instability of the individual's experiences. Pathological gamblers live their lack of balance in gambling, organizing their experience in such a way as to move their sense of void and lack of safety into compulsion. The group, by giving balance to the disorder instability, tends to progressively stop the seesaw trend that characterizes this typology of patients. Gambling in itself is a source of legitimate liking and is deeply rooted in the history and culture of any people, to the extent that it cannot be forbidden tout court. However, it may take on a compulsive and destructive character because of dependence processes and models that are similar, in certain respects, to the most known ones of alcoholism and drug addiction. ${ }^{1}$ In order to identify the indicators of gambling disorder, first of all it is necessary to distinguish between pathological gamblers and non-pathological gamblers based on the criterion of liking: this is related to gambling itself rather than to money from winning, as in drug addition, where the reward is just the fever, the flash that drug is able cause. This is a distinctive feature between non-pathological gamblers, who play to win, and pathological gamblers, who play to continue playing.

Gambling disorder is a secondary consequence of a voluntary persistent gambling behaviour in an individual that is vulnerable to dependence, i.e. with pre-existing neurofunctional alterations in some neurobiological systems. Amongst the systems concerned: the reward system (dopaminergic reward with anomalous hyper-response to gambling), the impulse control system (prefrontal cortex with selfcontrol deficit) and related cognitive functions (cognitive beliefs and distortions with reference to real chances of winning). According to the cognitive-behavioural approach, predisposing factors that make gambling pathological include: search for strong sensations that counter dysregulated emotions, desire to win money accompanied by a misconception of chance trends and, last but not least, remembering gambling-related positive events differently compared to negative ones. Indeed, gamblers remember wins arising their arousal and forget losses (Dickerson, idem). Given the disorder complexity, an approach is useful that implies intervention modes on several fronts, involving also the patient's relatives, if possible. From the moment the gambler contacts the Ser.T until their taking over, the first question to ask in the assessment stage relates to the seriousness of the situation, through clinical interviews and specific diagnostic tools: MMPI-2-Minnesota Multiphasic Personality Inventory, SOGS-South Oaks Gambling Screen for adults. GABS-Gambling Attitudes and Belief Survey. ${ }^{2}$
It will be useful to investigate the presence of any comorbidity and, once the patient has decided to undergo the treatment, it will be necessary to establish a therapeutic contract that includes the agreement to the cure and investigates the patient's expectations with reference to changes implied by the process. Within the group therapeutic cycle including the patient, the treatment goals will be:

i. Increasing the patient's awareness of their psychic functioning

ii. Recognizing and managing desire and impulses

iii. Favouring change

iv. Helping the patient recognize risks and obstacles

v. Developing personal defence strategies

vi. Increasing problem-solving capacities

\section{vii. Monitoring and assessing progresses and changes}

In order to increase the patient's awareness of their psychic functioning, they will comply with the group's rules by telling their personal experiences and issues related to gambling. The therapist will invite the patient to reflect upon and verbalize their thoughts, emotions and reactions until that moment and assess the possible range of alternatives. In the circle time, each patient will be able, in turns, to tell about themselves by filling in a worksheet to write down in the "table of functional analysis" the events characterizing the beginning of the dysfunctional behaviour, associated thoughts and sensations, behaviours and positive and negative consequences.

Since the goal of this treatment stage is to know one's own desire to gamble, the second worksheet will provide some hints for the group to work on:

i. Understanding the meaning of compulsion by collecting personal data on experiences, attitudes and behaviours related to compulsivity;

ii. Recognizing desire and impulse, by writing down situations in which desire appears and describe it when it becomes impulse

iii. Desire as "normal" experience

iv. How to face impulse: fighting sensations of need, personal experiences of managing desires and impulses

v. Functional strategies: directing thought, taking one's mind off things, being involved in some activities, personal defence strategies, which gambling-related desires/impulses emerge in each participant and strategies used.

In the subsequent worksheet, the patient will write down their spontaneous thoughts related to gambling when they emerge and, therefore, the defence strategy used. In their self-exploration process, the patient shall recognize or, more often, reconstruct internal and external reasons:

\section{i. Why is gambling a problem for me?}

ii. Why have I decided to give it up?

iii. Advantages and disadvantages of giving up and continuing

Afterwards, in the subsequent meetings, a sheet/guide of shortterm goals will be provided and, later, another one related to longterm goals, in which the patient shall write down: 
i. The changes I want to make in the next 12 weeks/12 months are...

ii. The most important reasons for which I want to make these changes are...

iii. The steps necessary for each change are...

iv. The ways other people can help me are...

v. The things that may interfere with my plans are....

A priority will be identified and given to the goals through the analysis of obstacles to their achievement, thanks to strategies aimed at keeping motivation high.

Afterwards, there will be an assessment of personal strategies of abstention from gambling by examining sources that stir impulses, measures to be taken to weaken them, monitoring of gambling-related behaviours all this always by filling in worksheets. In favouring a choice by the patient, the patient accustoms to assessing the safe alternative vs the risky one and using behavioural techniques for a functional assessment of risk. At the end of it, in the subsequent meetings, there will be exercises on risk situations, with a series of alternative solutions and resources to be taken into account, followed by an analysis of skills achieved and an assessment of personal processes implemented. Life goals will be re-adjusted, finally including the support plan, in which the patient shall write down, in a grid, a hierarchy of goals crossing the help source for their achievement, times, help modes and results achieved.

\section{Conclusions}

The experience of the group treatment, by creating temporary group identification, allows individuals to get from the group an identity share that the gambling disorder itself contributes to spread. This way, the aware use of planned strategies and the sharing of common issues make it possible to use the group treatment for compulsive gambling as one of the most effective resources the State may invest in so that Ser.Ts become an increasingly visible point of reference for the treatment and cure of "behavioural dependencies".

\section{Acknowledgments}

I would like to thank Dr Giuseppe Anastasi, Head Psychologist of the Ser.T of Ostia Lido for his support in the co-management of the group during the treatment.

\section{Conflicts of interest}

I hereby declare that there are no conflicts of interest.

\section{References}

1. Dickerson MG. La Dipendenza da Gioco. Edizioni Gruppo Abele, Torino. 1993.

2. American Psychiatric Association (APA). Diagnostic and Statistical Manual of Mental Disorders, 5th ed. In: Artington VA, editor. Italian translation Criteri diagnostici Mini DSM-5, Raffaello Cortina Editore, Milano. 2014. 